\title{
Influencia de dos tipos de fertilización sobre la flora adventicia, la producción y el contenido en flavonoides en un cultivo de Achillea millefolium L. en Galicia (NW de España).
}

\author{
Recibido: 31 Maio 2007 / Aceptado: 4 Outubro 2007 \\ (c) IBADER- Universidade de Santiago de Compostela 2007
}

\begin{abstract}
Resumen Achillea millefolium L. (milenrama) es una de las plantas medicinales más demandadas en los mercados internacionales. Paralelamente a su interés económico ha crecido la superficie dedicada a su cultivo, aunque las prácticas agronómicas relacionadas con la optimización del mismo están todavía poco desarrolladas. Aspectos que deben ser investigados se refieren a la fertilización y al control de las malas hierbas por métodos no químicos, ya que el objetivo de cultivos de este tipo es la obtención de principios activos para formulaciones libres de residuos químicos. En este trabajo se estudió la influencia de dos tipos de abonado (mineral y orgánico) sobre la producción de Achillea millefolium y su contenido en flavonoides, controlando las poblaciones arvenses mediante escarda manual, durante el primer año de cultivo. Se ha comprobado que si no se emplean fertilizantes, las escardas manuales controlan eficazmente las adventicias. El abonado (tanto orgánico como mineral) incrementó significativamente la diversidad, cobertura y frecuencia de la flora adventicia, y las escardas manuales no fueron efectivas. Así, la mayor producción de biomasa de planta medicinal, tanto de cabezuelas florales como de parte vegetativa, se produjo en las parcelas no fertilizadas. Se comprobó que las producciones obtenidas en las parcelas fertilizadas con abono orgánico fueron significativamente superiores a las obtenidas en las de fertilización inorgánica. Los distintos tratamientos de fertilización no repercutieron significativamente sobre la producción de flavonoides, si
\end{abstract}

Rosa Romero - Ma Jesús Saínz · Antonio Rigueiro - Ma Pilar González-Hernández · $M^{\mathrm{a}}$ Elvira López-Mosquera Departamento de Produción Vexetal, Escola Politécnica Superior, Universidade de Santiago de Compostela. Edificio administrativo da Escola Politécnica Superior de Lugo. E-27002 Lugo, España e-mail: rosarome@lugo.usc.es bien la fertilización, sobre todo la mineral, tuvo efectos positivos sobre la aparición de este tipo de compuestos en los tallos. Estos resultados pusieron de manifiesto que el control de las adventicias es fundamental para optimizar la producción de Achillea millefolium en cultivo. Las técnicas culturales empleadas pueden también influir en la calidad de los principios activos.

Palabras clave Achillea millefolium L. milenrama, fertilización orgánica, fertilización mineral, principios activos.

Abstract In recent years, yarrow cultivation (Achillea millefolium L.) has been increasing in several European countries, in view of increasing demand from the herbal medicine and cosmetics industries. However, little is known about optimal cultivation practices for this crop, such as fertilization and weed control. Weed control is of particular interest, since buyers generally require the crop to be free of herbicide residues. In the present study we investigated the effects of fertilizer use (mineral fertilizer, organic fertilizer, or no fertilizer) on weed flora and the yield and flavonoid contents in a first-year yarrow crop in northwest Spain. Weeds were controlled by manual hoeing three times over the 10-month cultivation period. Our results indicate that neither mineral nor organic fertilization were beneficial: both types of fertilization increased weed cover and weed species diversity, and significantly reduced yarrow yield. In the non-fertilized plots, by contrast, weed cover was maintained at acceptable levels by the manual hoeing. Furthermore, fertilization did not appear to improve flavonoid contents, except for a small effect on apolar flavonoid contents in stems with mineral fertilization. These results suggest that fertilization is not beneficial for first-year yarrow crops in our region, though this conclusion may not be generalizable to other regions.

Key words: Achillea millefolium L., yarrow, NW Spain, organic fertilization, mineral fertilization. 


\section{Introduccion}

La milenrama, Achillea millefolium L. es una planta perenne perteneciente a la familia Asteraceae, autóctona en Europa y Asia y naturalizada en América del Norte. Desde la antigüedad se utiliza como planta medicinal por sus propiedades tónicas, aperitivas, digestivas, eupépticas, antiespasmódicas, hemostáticas, emenagogas, antisépticas y antiinflamatorias (Chandler et al. 1982). Las investigaciones tendentes a conocer los principios activos responsables de las distintas acciones terapéuticas comenzaron hace más de 200 años, existiendo en la actualidad un amplio conocimiento sobre algunos de sus componentes y su modo de actuación (Hoffman, 1719; Falk, et al.; 1975; Chandler et al, 1982; Mitich, 1990; Figueiredo et al. 1992; Hoffman et al 1992; Afsharypour et al.; 1996; Lourenço et al., 1999). Así, se ha puesto de manifiesto que la milenrama contiene una gran variedad de compuestos, tales como aceite esencial, alcaloides terpenoides, lactonas sesquiterpénicas, taninos y flavonoides. Estos últimos han cobrado gran interés en los tiempos recientes debido a sus múltiples efectos sobre la salud: prevención de dolencias circulatorias y osteoporosis (Wang et al.,1994), acción antivírica (Vrifsen et al., 1988), acción antiparasitaria (Mitra et al, 2000, acción anticancerosa (Yoshida et al., 1990), acción antiinflamatoria (Kin et al., 1998) y acción antioxidante (Brown \& Rice-Evans, 1998, Pérez et al 2000,). Por ello, las plantas que, como la milenrama, los producen en abundancia son muy demandadas por parte de la industria farmacéutica, cosmética, alimentaria y parafarmacéutica. Con el fin de abastecer el mercado con productos de calidad se está promoviendo el cultivo de esta especie en distintas zonas del mundo, lo que hace necesarias investigaciones encaminadas a optimizar su rendimiento. Se han realizado algunos estudios sobre la influencia de la fertilización y de la humedad del suelo en la producción de planta y de aceite esencial (El-Kholy, 1984; Scheffer et al., 1993).

Según indicaciones de los productores de planta medicinal, un aspecto importante que debe ser investigado es el control de las malas hierbas por métodos no químicos, ya que el objetivo de este tipo de cultivos suele ser la obtención de principios activos libres de residuos de fitocidas.

En este trabajo se estudió la influencia de dos tipos de abonado (mineral y orgánico) sobre la producción de Achillea millefolium y su contenido en flavonoides, durante el primer año de cultivo, en una región del NW de España. Asimismo se evaluó la aparición de malas hierbas en los distintos tratamientos.

\section{Material y métodos}

El ensayo se realizó en una explotación dedicada al cultivo de plantas medicinales situada en Galicia ( NW de España) ( $7^{\circ} 56^{\prime} \mathrm{W}-42^{\circ} 53^{\prime} \mathrm{N}$ ), a $470 \mathrm{~m}$ de altitud. En la zona la temperatura media anual fue de $11,9{ }^{\circ} \mathrm{C}$ y la precipitación media anual de $1305 \mathrm{~mm}$. El suelo sobre el que se llevó a cabo el ensayo es un Cambisol eutrico desarollado sobre las serpentinitas de Melide (Buurman, 1985).
El análisis químico del suelo de la zona en la que se estableció el ensayo se presentan en la Tabla 1 destacando los altos niveles de calcio y los bajos de fósforo y potasio.

Para el desarrollo del ensayo se establecieron doce parcelas de $8 \mathrm{~m}^{2}$ cada una, separadas por pasillos de 1,5 m, en las que se aplicaron tres tratamientos de fertilización en un diseño completamente al azar (cuatro réplicas por tratamiento): control, mineral y orgánico. En las parcelas control no se realizó ningún abonado. La fertilización mineral consistió en la aplicación de $667 \mathrm{~kg}$ ha ${ }^{-1}$ de abono 15.15.15 y la orgánica en la incorporación de $4000 \mathrm{~kg}$ ha-1 de un abono a base de estiércol de pollo deshidratado y granulado que contenía un $4 \%$ de $\mathrm{N}$; un $2,2 \%$ de $\mathrm{K}_{2} \mathrm{O}$ y un $2,6 \%$ de $\mathrm{P}_{2} \mathrm{O}_{5}$. Los fertilizantes se aplicaron antes de efectuar la plantación.

La plantación se realizó en septiembre de 2002 con plántulas silvestres homogéneas, de unos $8 \mathrm{~cm}$ y con seis hojas desarrolladas, procedentes de las inmediaciones de la zona de cultivo. La separación entre líneas fue de $50 \mathrm{~cm}$ y la de plantas dentro de cada línea de $30 \mathrm{~cm}$.

Durante el desarrollo del cultivo se realizaron tres escardas manuales (febrero, marzo y abril de 2003) en todas las parcelas y se inventariaron las especies botánicas adventicias que fueron apareciendo en los distintos tratamientos, siguiendo el método descrito por Maillet (1981). En cada parcela se estimaron los siguientes parámetros: cobertura total de adventicias (\%), índice de abundancia de Barralis (1976), frecuencia relativa (\%), tipo biológico (Raunkaier, 1905) y biomasa de malas hierbas (determinación del peso seco mediante secado en estufa de aire forzado a $60^{\circ} \mathrm{C}$ durante 48 horas.).

A mediados de junio se procedió a la recolección de la parte aérea de la planta y se estimó su peso seco después de secado en estufa a $30^{\circ} \mathrm{C}$ (temperatura de estabilización de los compuestos medicinales), cuantificándose por separado la producción de cabezuelas florales y de tallo con hojas.

En submuestras homogéneas de ambas fracciones se determinó el contenido en flavonoides totales y apolares mediante la técnica de HPLC (en un equipo JASCO automático). Para ello $100 \mathrm{mg}$ de cada muestra (por duplicado) se sometió a una extracción con methanol-agua usando ultrasonidos durante 5 minutos. Este proceso se repitió tres veces. El sobrenadante fué recogido y ajustado hasta un volume de $25 \mathrm{ml}$. Extractos de $25 \mu \mathrm{l}$ fueron eluídos a través de una columna RP-18 (5 um $\mathrm{C}_{18}, 150 \times 4,60$, Phenomenex).

La fase móvil fue acetonitrilo metanol/tampón fosfato $5 \mathrm{mM}$ $\mathrm{pH} 2,55$. El flujo fue de $1,0 \mathrm{ml} / \mathrm{min}$ y la longitud de onda de $335 \mathrm{~nm}$. Los patrones internos utilizados fueron apigenina $(0,2 \mathrm{mg} / \mathrm{ml})$ y apigenina-7-glucósido $(0,05 \mathrm{mg} / \mathrm{ml})$ disueltos en etanol al $70 \%$ (Schulz \& Albroschert).

El tratamiento estadístico se realizó por comparación de medias a través de un análisis de varianza con un solo factor, comprobando previamente si los datos eran normales (prueba de Kolmogorov-Smirnov) y realizando la prueba de homogeneidad de la varianza de Levene. Se utilizó el paquete estadístico SPSS (2004). 


\begin{tabular}{|c|c|c|c|c|c|c|c|c|c|c|c|c|}
\hline $\begin{array}{l}\mathrm{pH} \\
\mathrm{H}_{2} \mathrm{O} \\
\end{array}$ & $\begin{array}{l}\mathrm{pH} \\
\mathrm{CIK}\end{array}$ & $\% \mathrm{C}$ & $\% \mathrm{MO}$ & $\% \mathrm{~N}$ & $\mathrm{C} / \mathrm{N}$ & $\begin{array}{l}\mathrm{mg} / \\
\mathrm{kg} \mathrm{P}\end{array}$ & $\begin{array}{l}\mathrm{Cmol}(+) / \\
\mathrm{kg} \mathrm{Ca}\end{array}$ & $\begin{array}{l}\mathrm{Cmol}(+) / \\
\mathrm{Kg} \mathrm{Mg} \\
\end{array}$ & $\begin{array}{l}\mathrm{Cmol}(+) / \\
\mathrm{kg} \mathrm{Na}\end{array}$ & $\begin{array}{l}\mathrm{Cmol}(+) \mathrm{l} \\
\mathrm{kg} \mathrm{K}\end{array}$ & $\begin{array}{l}\mathrm{Cmol}(+) / \\
\mathrm{kg} \mathrm{Al}\end{array}$ & $\begin{array}{l}\text { \%sat } \\
\mathrm{Al}\end{array}$ \\
\hline 5,70 & 5,30 & 3,88 & 6,71 & 0,244 & 37,5 & 6,37 & 5,34 & 0,69 & 0,78 & 0,29 & 0,38 & 5,03 \\
\hline
\end{tabular}

Tabla 1.- Análisis químico del suelo del área de estudio

\section{Resultados y discusion}

\section{Producción de Achillea millefolium}

Como se puede observar en la Tabla número 2, la mayor producción de biomasa de planta medicinal, tanto de cabezuelas florales como de parte vegetativa, se produjo en las parcelas no fertilizadas.

Estos resultados, contrarios a los que en un principio serían esperables y no coincidentes con lo publicado por algunos autores (El-Kholy, 1983, Scheffer et al, 1993), seguramente estén relacionados con la menor incidencia de plantas infestantes en las parcelas no fertilizadas (tal como se verá más adelante) y con el hecho de que, al ser la achillea una planta rústica, poco exigente en nutrientes, la fertilidad del suelo sin fertilizar puede ser suficiente para satisfacer sus demandas nutritivas, al menos durante el primer año de cultivo.
Si comparamos los dos tipos de fertilización entre si observamos que fueron más productivas las parcelas fertilizadas con el abono orgánico, que no difieren significativamente de las parcelas control pero si de las tratadas con abono inorgánico. Esta tendencia es similar a la encontrada en otros cultivos realizados en el área de estudio, comparando el efecto de ambos tipos de abonado (López-Mosquera et al, 2003), y parece estar más relacionada con el efecto beneficioso de las enmiendas orgánicas sobre las propiedades físicas del suelo y sobre su actividad biológica que con la incorporación inmediata de nutrientes al mismo.

Estos argumentos quedan avalados cuando se compara la evolución de los macronutrientes (nitrógeno, fósforo y potasio) en los tres tratamientos durante el ciclo del cultivo, que va desde el momento de la incorporación del abonado y plantación hasta la recogida de la biomasa producida (Tabla 3).

\begin{tabular}{lllll}
\hline Tratamiento & $\begin{array}{l}\text { Peso fresco } \\
\text { de flor }\end{array}$ & $\begin{array}{l}\text { Peso seco } \\
\text { de flor }\end{array}$ & $\begin{array}{l}\text { Peso fresco } \\
\text { de tallo }\end{array}$ & $\begin{array}{l}\text { Peso seco } \\
\text { de tallo }\end{array}$ \\
\hline Control & $496,9 \pm 78,4 \mathrm{a}$ & $159,5 \pm 46,8 \mathrm{a}$ & $603,7 \pm 92,5 \mathrm{a}$ & $225,8 \pm 29,6 \mathrm{a}$ \\
Mineral & $157,1 \pm 50,2 \mathrm{~b}$ & $45,0 \pm 14,0 \mathrm{~b}$ & $250,5 \pm 84,8 \mathrm{~b}$ & $84,2 \pm 25,7 \mathrm{~b}$ \\
Orgánico & $422,5 \pm 49,5 \mathrm{a}$ & $111,6 \pm 0,9 \mathrm{a}$ & $587,5 \pm 29,0 \mathrm{a}$ & $201,6 \pm 10,9 \mathrm{a}$ \\
\hline
\end{tabular}

Tabla 2.- Media y desviación típica del peso fresco y seco (kg ha-2) en flor y tallos con hojas de Achillea millefolium. Los datos seguidos de distinta letra son significativamente diferentes para $p<0,05$

\begin{tabular}{|c|c|c|c|c|c|c|c|c|c|}
\hline & \multicolumn{3}{|c|}{ Control } & \multicolumn{3}{|c|}{ Organic } & \multicolumn{3}{|c|}{ Mineral } \\
\hline & & g) $K(\mathrm{mg} / \mathrm{kg})$ & $\% N$ & $\mathrm{P}(\mathrm{mg} / \mathrm{kg}$ & $\mathrm{K}(\mathrm{mg} / \mathrm{kg})$ & $\% \mathrm{~N}$ & $P(n$ & $\mathrm{K}(\mathrm{mg} / \mathrm{kg})$ & $\% \mathrm{~N}$ \\
\hline Sep 2002 & 6.37 & 0.29 & 0.32 & 13.99 & 0.57 & 0.44 & 14.12 & 0.51 & 0.43 \\
\hline Mar 2003 & 2.98 & 0.25 & 0.24 & 4.83 & 0.30 & 0.21 & 5.33 & 0.47 & 0.23 \\
\hline Jul 2003 & 1.43 & 0.25 & 0.17 & 2.833 & 0.19 & 0.19 & 2.84 & 0.32 & 0.17 \\
\hline
\end{tabular}

Tabla 3.- Niveles de macronutrientes en el suelo

\section{Contenido en flavonoides}

Las distintas industrias que utilizan sustancias antioxidantes están restringiendo el uso de los compuestos sintéticos a favor de los antioxidantes naturales con el fin de evitar o reducir los efectos secundarios (Veliglu et al., 1998). Así se entiende que en los últimos años numerosas investigaciones se hayan encaminado en la búsqueda de fuentes naturales de este tipo de productos (Veliglu et al., 1998; Gazzani et al., 1998; Larson, 1998; Vinson et al., 1988; Cao et al., 1996). Entre estas posibles fuentes naturales se encuentran distintas especies del género Achillea, de las que ya se ha constatado experimentalmente su capacidad antioxidante (Candan et al., 2003, Souri et al., 2004).
Teniendo en cuenta estos antecedentes se ha creído oportuno analizar la influencia de los distintos tratamientos culturales ensayados sobre el contenido en flavonoides de la especie estudiada. Puesto que las formulaciones glicosídicas son menos efectivas, debido a su polaridad, que sus agliconas ( Rice-Evans et al, 1996, Cao et al, 1997), se ha considerado interesante conocer también el contenido de este último tipo de compuestos.

Tal como se muestra en la Tabla 4, la milenrama produce fundamentalmente los flavonoides en combinaciones glicosídicas, siguiendo el patrón que parece ser el usual en la naturaleza (Cao et al, 1977, Wolenweber, 1988). La parte de la planta que ofrece un mayor rendimiento es la flor. Los flavonoides apolares, aunque minoritarios, y al contrario que la fracción polar, se producen casi exclusivamente en las cabezuelas florales. De hecho, el tratamiento estadístico no 


\begin{tabular}{lllll}
\hline Tratamiento & $\begin{array}{l}\text { Flavonoides totales } \\
\text { en flor }(\%)\end{array}$ & $\begin{array}{l}\text { Flavonoides totales } \\
\text { en tallo }(\%)\end{array}$ & $\begin{array}{l}\text { Aglyconas } \\
\text { en flor }(\%)\end{array}$ & $\begin{array}{l}\text { Aglyconas } \\
\text { en tallo(\%) }\end{array}$ \\
\hline Control & $0,18 \pm 0.005 \mathrm{a}$ & $0,13 \pm 0,03 \mathrm{a}$ & $0,01 \pm 0,001 \mathrm{a}$ & $0,0004 \pm 0.0003 \mathrm{a}$ \\
Mineral & $0,18 \pm 0.009 \mathrm{a}$ & $0,15 \pm 0.03 \mathrm{a}$ & $0,01 \pm 0.001 \mathrm{a}$ & $0,0009 \pm 0.0001 \mathrm{~b}$ \\
Orgánico & $0,18 \pm 0.005 \mathrm{a}$ & $0,12 \pm 0,01 \mathrm{a}$ & $0,01 \pm 0.001 \mathrm{a}$ & $0,0006 \pm 0.0002 \mathrm{ab}$ \\
\hline
\end{tabular}

Tabla 4.- Media y desviación típica de la proporción de flavonoides totales y aglyconas en flores y tallos con hojas de Achillea millefolium en su primera cosecha y en los distintos tratamientos. Para cada columna, los datos seguidos de distinta letra son significativamente diferentes para $p<0,05$

revela diferencias significativas cuando se comparan las cantidades de flavonoides polares producidos en el tallo con los producidos en la flor (para cada tratamiento de fertilización), mientras que si existen cuando se comparan las aglyconas, lo que es importante pues la fracción comercializada suele ser la floral.

Los distintos tratamientos de fertilización no repercuten significativamente sobre la producción de flavonoides, si bien parece que la fertilización, sobre todo la mineral, tiene efectos positivos sobre la aparición de este tipo de compuestos en los tallos, sobre todo en lo concerniente a las aglyconas que llegan a duplicar su valor en este tratamiento. Estos resultados parecen ir en la línea de que el contenido de componentes activos en Achillea millefolium, depende no sólo de factores genéticos, sino también ambientales, entre los que habría que incluir la fertilización (Verne, 1981, Maffei et al, 1994).

Flora adventicia desarrollada en el cultivo de Achillea

En las parcelas fertilizadas, tanto con el fertilizante mineral como con el orgánico, el número de especies adventicias fue significativamente mayor que las parcelas control (tabla 5).

En las parcelas fertilizadas con abono mineral se encontraron 46 especies pertenecientes a 16 familias botánicas. En las tratadas con abono orgánico 38 especies, correspondientes a 15 familias. Mientras que en las parcelas control sólo se inventariaron 22 especies de 12 familias. En todos los tratamientos las familias más representadas fueron Poaceae, Asteraceae y Fabaceae (Tabla 6), coincidiendo con otros estudios realizados en diversos cultivos dentro de la misma área biogeográfica (Buján et al. 1995; Fernández et al, 1994), lo que podría explicarse por la importante representación de estas familias en la flora atlántica y por la aptitud de muchas de sus especies para adaptarse a medios inestables (Maillet, 1981; Gillerm and Maillet, 1982).

En las parcelas no fertilizadas fue menor el número de especies y también el índice de abundancia de éstas, pues ninguna superó los 3 individuos por $\mathrm{m}^{2}(\mathrm{la}=2)$. En cambio en las parcelas fertilizadas algunas especies presentaron índices de abundancia y de frecuencia elevados (la =3-4) (Tabla 5). Además, en éstas últimas parcelas aparecen numerosas especies que, aunque poco abundantes, contribuyen a enriquecer las comunidades estacionales de malas hierbas y suponen un riesgo de infestación a corto plazo (Tabla 6).
El abonado también incrementó significativamente la cobertura de las especies adventicias, superior al $90 \%$ en las parcelas abonadas frente al $10 \%$ de las parcelas control. El mismo comportamiento se observó cuando se analizaron los datos referentes a la biomasa total de las malas hierbas, pues en las parcelas no fertilizadas es la sexta parte de la de las abonadas (Tabla 7).

El desarrollo de las comunidades de adventicias está influído por el manejo dado al cultivo, fundamentalmente por el método de laboreo y la fertilización aplicada (Sommers, 1985; Barberi et al., 1997; McClosky et al., 1998; Feldman et al., 1998; Pino et al., 1998; Zhlekova, 2001). La incorporación de nutrientes al suelo mediante fertilización repercute positivamente tanto sobre el crecimiento de los cultivos como sobre la flora adventicia (Barberi et al., 1998; Mcklosky et al., 1998; Barret \& Campbel, 1973; González Ponce, 1998). Parece por tanto, que las escardas manuales efectuadas son suficientes para controlar las adventicias en las parcelas no fertilizadas pero insuficientes en las fertilizadas. Autores como Barberi et al. (1988) y Feldman et al. (1997) indican que las escardas manuales conllevan un incremento del banco de semillas en la capa superficial del suelo, que indudablemente se ven favorecidas con la fertilización.

Las plantas anuales fueron dominantes en los tres tratamientos (Tabla 8), pero la mayoría de las especies más infestantes, y potencialmente más peligrosas para el cultivo, es decir aquellas que presentan mayores abundancias y frecuencias, son plantas vivaces, aspecto que parece estar relacionado con la ausencia de labores profundas (Taleb, 1989; Taleb \&Maillet, 1994). La Tabla 5 muestra que la fertilización provoca la dominancia de unas pocas especies, fundamentalmente vivaces (Holcus mollis, Hypochoeris radicata, Briza media, Rumex acetosa), aspecto muy negativo de cara al manejo posterior de la flora arvense, puesto que algunas de ellas una vez instaladas son muy difíciles de erradicar (Liebman y Dick, 1993).

\section{Conclusiones}

Estos resultados indican que el tipo de fertilización empleado tiene repercusión en la producción y calidad de la planta medicinal.

Se pone de manifiesto la necesidad de un control efectivo de la flora adventicia asociada al cultivo de la Achillea millefolium, puesto que las malas hierbas no sólo reducen la producción, sino que además dificultan la cosecha y pueden producir contaminación de los principios activos. 


\begin{tabular}{|c|c|c|c|c|c|c|c|c|}
\hline \multicolumn{3}{|l|}{ Control } & \multicolumn{3}{|l|}{ Orgánico } & \multicolumn{3}{|l|}{ Mineral } \\
\hline Especies & la & $\mathrm{Fr}$ & Especies & la & $\mathrm{Fr}$ & Especies & la & $\mathrm{Fr}$ \\
\hline Holcus mollis & 2 & 100 & Holcus mollis & 4 & 100 & Holcus mollis & 4 & 100 \\
\hline Raphanus raphanistrum & 2 & 100 & Rumex acetosa & 4 & 100 & Hypochoeris radicata & 3 & 100 \\
\hline Hypochoeris radicata & 1 & 100 & Briza media & 3 & 100 & Poa annua & 3 & 100 \\
\hline Poa annua & 1 & 100 & Poa annua & 3 & 100 & Rumex acetosa & 3 & 100 \\
\hline Lotus corniculatus & 1 & 100 & Anthemis arvensis & 3 & 75 & Anthemis arvensis & 3 & 75 \\
\hline Rumex acetosa & 1 & 75 & Briza maxima & 3 & 75 & Parentucellia viscosa & 2 & 75 \\
\hline Anthemis arvensis & 1 & 50 & Parentucellia viscosa & 3 & 75 & Anthoxantum odoratum & 2 & 50 \\
\hline Trifolium pratense & 1 & 25 & Raphanus raphanistrum & 3 & 50 & Cerastium glomeratum & 2 & 50 \\
\hline Sonchus oleraceus & 1 & 25 & Avena sterillis & 2 & 100 & Raphanus raphanistrum & 2 & 50 \\
\hline Anagallis arvensis & 1 & 25 & Lotus corniculatus & 2 & 100 & Campanula patula & 1 & 100 \\
\hline Hypericum humifisum & 1 & 25 & Geranium robertianum & 2 & 100 & Lotus corniculatus & 1 & 100 \\
\hline Ulex europaeus & 1 & 25 & Veronica persica & 2 & 100 & Ornithopus perpusillus & 1 & 100 \\
\hline Arenaria serpyllifolia & 1 & 25 & Hypochoeris radicata & 2 & 75 & Vulpia bromoides & 1 & 75 \\
\hline Dactylis glomerata & 1 & 25 & Andryala integrifolia & 2 & 50 & Andryala integrifolia & 1 & 50 \\
\hline Aira praecox & 1 & 25 & Calamintha nepeta & 2 & 50 & Arenaria serpillifolia & 1 & 50 \\
\hline Agrostis stolonifera & 1 & 25 & Leontodon taraxacoides & 2 & 50 & Briza media & 1 & 50 \\
\hline Veronica persica & 1 & 25 & Spergula arvensis & 2 & 50 & Cytisus striatus & 1 & 50 \\
\hline Parentucellia viscosa & 1 & 25 & Stellaria media & 2 & 50 & Spergularia arvensis & 1 & 50 \\
\hline Polygala vulgaris & 1 & 25 & Trifolium pratense & 2 & 50 & Stellaria media & 1 & 50 \\
\hline Fallopia convolvulus & 1 & 25 & Anagallis arvensis & 2 & 25 & Trifolium pratense & 1 & 50 \\
\hline Plantago lanceolata & 1 & 25 & Cytisus striatus & 2 & 25 & Veronica persica & 1 & 50 \\
\hline Jasione montana & 1 & 25 & Lithodora prostrata & 2 & 25 & Briza maxima & 1 & 25 \\
\hline & & & Aphanes arvensis & 1 & 25 & Conyza canadensis & 1 & 25 \\
\hline & & & Cerastium glomeratum & 1 & 50 & Dactylis glomerata & 1 & 25 \\
\hline & & & Daboecia cantabrica & 1 & 25 & Prunella vulgaris & 1 & 25 \\
\hline & & & Dactylis glomerata & 1 & 25 & Leontodon taraxacoides & 1 & 25 \\
\hline & & & Fallopia convolvulus & 1 & 50 & Ulex europaeus & 1 & 25 \\
\hline & & & Fumaria officinalis & 1 & 25 & Sonchus oleraceus & 1 & 25 \\
\hline & & & Gladiolus illyricus & 1 & 50 & Aphanes arvensis & 1 & 25 \\
\hline & & & Hypericum humifusum & 1 & 25 & Anagallis arvensis & 1 & 25 \\
\hline & & & Iberis amara & 1 & 25 & Silene gallica & 1 & 25 \\
\hline & & & Ornithopus perpusillus & 1 & 25 & Hypericum humifusum & 1 & 25 \\
\hline & & & Reseda phyteuma & 1 & 25 & Vulpia bromoides & 1 & 25 \\
\hline & & & Rubus sp. & 1 & 25 & Senecio vulgaris & 1 & 25 \\
\hline & & & Silene gallica & 1 & 25 & Stellaria media & 1 & 25 \\
\hline & & & Ulex europaeus & 1 & 25 & Aira praecox & 1 & 25 \\
\hline & & & Vulpia bromoides & 1 & 25 & Agrostis stolonifera & 1 & 25 \\
\hline & & & Sherardia arvensis & 1 & 25 & Lupinus sp. & 1 & 25 \\
\hline & & & & & & Andryala integrifolia & 1 & 25 \\
\hline & & & & & & Vicia sp. & 1 & 25 \\
\hline & & & & & & Sanguisorba minor & 1 & 25 \\
\hline & & & & & & Sherardia arvensis & 1 & 25 \\
\hline & & & & & & Plantago lanceolata & 1 & 25 \\
\hline & & & & & & Fumaria officinalis & 1 & 25 \\
\hline & & & & & & Rubus sp. & 1 & 25 \\
\hline & & & & & & Reseda lutea & 1 & 25 \\
\hline
\end{tabular}

Fr: Frecuencia relativa (\%), Ia: Abundancia media: $1=1$ ind. $\mathrm{m}^{-2} ; 2=1$ a 3 ind. $\mathrm{m}^{-2} ; 3=4$ a 20 ind. $\mathrm{m}^{-2}$; $4=21$ a 50 ind. $\mathrm{m}^{-2} ; 5=>50$ ind. $\mathrm{m}^{-2}$

\begin{tabular}{lcccccc}
\hline Familias & Poaceae & Asteraceae & Fabaceae & Polygonaceae & Scrophulariaceae & Caryophyllaceae \\
\hline Control & 20,0 & 16,0 & 16,0 & 12,0 & 8,0 & 4,0 \\
\hline Orgánico & 17,0 & 12,5 & 12,5 & 5,0 & 5,0 & 10,5 \\
\hline Mineral & 22,7 & 15,0 & 13,6 & 2,2 & 6,8 & 11,3 \\
\hline
\end{tabular}

Tabla 5.- Catálogo florístico de las malas hierbas presente en los distintos tratamientos

Tabla 6.- Familias más representados y $\%$ de especies adventicas en cada tratamiento pertenecientes a cada una de ellas

\begin{tabular}{ccc}
\hline Tratamiento & Biomasa $\left(\mathrm{g} / \mathrm{m}^{2}\right)$ & Cobertura $(\%)$ \\
\hline Control & $4,97 \pm 1,5 \mathrm{a}$ & $10 \mathrm{a}$ \\
Orgánico & $31,40 \pm 5,9 \mathrm{~b}$ & $93 \mathrm{~b}$ \\
Mineral & $29,01 \pm 6,3 \mathrm{~b}$ & $90 \mathrm{~b}$ \\
\hline
\end{tabular}

Tabla 7.- Media y desviación típica de la biomasa y cobertura de las malas hierbas en los distintos tratamientos aplicados. Para cada columna los datos seguidos de distinta letra son significativamente diferentes para $p<0,05$

\begin{tabular}{lccc}
\hline tratamiento & $\mathrm{T}$ & $\mathrm{H}$ & $\mathrm{C}$ \\
\hline control & 63,6 & 31,3 & 5,1 \\
Orgánico & 63,2 & 26,3 & 10,5 \\
Mineral & 74 & 21,7 & 4,3 \\
\hline
\end{tabular}

Tabla 8.- Tipos biológicos representados en la flora adventicia de cada tratamiento y proporción(\%) de especies correspondientes a cada uno. (T: Terófitos, H: Hemicriptófitos, C: Caméfitos) 
Agradecimientos Agradecemos a la Sociedade Cooperativa Galega Milhulloa la cesión de los terrenos en los que que se ha realizado el ensayo y a Cristina Vázquez y Susana Dopico por los trabajos técnicos. Este trabajo fue financiado por el proyecto PGIDIT029104PR.

\section{Bibliografía}

Afsharypour, S., Asgary, S. \& Lockwood, G.B. (1996). Volatile constituents of Achillea millefolium from Iran. Flavour Fragance Journal 11, 265-267.

Barberi, P., Silvestri, N. \& Bonari E. (1997). Weed communities of winter wheat as influenced by input level ant rotation. Weed Research 37, 301-314.

Barberi, P., Cozzani, A., Macchia, M., \& Bonari, E. (1998). Size and composition of weed seedbank under different management systems for continuous maize cropping. Weed Research 38, 319-334.

Barralis, G. (1976). Méthodes d'etude des groupements adventices des cultures annuelles, application à la Côte d'Or. In: Proceedings Véme Colloque Inernational sur l'Ecologie des mauvaises herbes, Dijon , 59-68.

Barret, D.W. \& Campbell, A. (1973). An evaluation of effects of competition between wheat and wimmera ryegrass (Lolium rigidum) durin early stages of growth. Australian Journal of Experimental Agriculture and Animal Husbandry. $13,581-585$.

Brown, J.E. \& Rice-Evans, C.A. (1998). Luteolin-rich artichoke extract protects low density lipoprotein from oxidaton "in vitro". Free Radical Research. 29, 247-255.

Buurman, P. (1985). Wheathering and soil forma in mafic and ultramafic rocks in N. Galicia, Spain. Department of Soil Science and geology. University Wageningen.

Castro, L.O. (1985). Informaçoes sobre a milfolhas (Achillea millefolium L.) e a sua cultura. Roesslèria 8, 65-68.

Buján, M., Castelao, A.M. \& Saínz, M.J. (1995). Malas hierbas en cultivo ecológico de espárrago (Asparagus officinalis L.) en Galicia: primeros resultados. In: Actas del Congreso 1995 de la Sociedad Española de Malherbología, Huesca (Spain), 83-86.

Chandler, R.F., Hoope,r S.N. \& Harvey, M.J. (1982). Ethnobotany and phytochemistry of yarrow, Achillea millefolium, Compositae. Economy Botany. 36, 203-223.

Cao, G., Sofic, E. \& Prior, R.L. (1997). Antioxidant and prooxidant behavior of flavonoids: structure-activity relantioships. Free Radical Biological of Medicine. 22, 749760.

Candan, F., Unlu, M., Tepe, B., Daferera, D., Polissiou, M., Sökmen, A. \& Akpulat, H.A. (2003). Antioxidant and antimicrobial activity of the essential oil and metanol extracts of Achillea millefolium subs millefolium Afan (Asteraceae). Journal of Ethnopharmacolog .87, 2, 215-220.

El-Kholy, S.A. (1984). Soil moisture and macronutrients effects on yield and quality of Achillea millefolium L. Minufiya Journal of Agriculture Research. 8, 331-348.
Falk, A.J., Smolenski, S.J., Bauer, L. \& Bell, C.L. (1975). Isolation and identification of three new flavones from Achillea millefolium L. Journal of Pharmaceutical Science. 64, 1838-1842.

Feldman, S.R., Alzugaray, C., Torres, P.S. \& Lewis, J.P. (1998). Gap colonization by weeds in wheat crop grown under different cultivation regimes. Weed Research. 38, 3546.

Fernández, J.L., Rigueiro, A. \& Romero, R. (1994). Populations de mauvaises herbes et leur contrôle dans des plantations de framboisier (Rubus idaeus L.) en Galicia. In: Proceedings 5 th EWRS Mediterranean Symposium, Perugia (Italy), 303-310.

Figueiredo, A.C., Barroso, J.G., Pais, M.S.S. \& Scheffer, J.J.C. (1992). Compositicon of the essential oil from leaves and flowers of Achillea millefolium ssp. millefolium.. Flavour Fragrance Journal. 7, 219-222.

Gazzani, G., Papetti, A., Massolini, G. \& Dagila, M. (1998). Anti and pro-oxidant activity of water soluble components of some common diet vegetables and the effect o thermal treatment. Journal Agricultural Food Chemistry. 46, 4118.4122.

González Ponce, R. (1998). Competition between barley and Lolium rigidum for nitrate. Weed Research. 38, 453-460.

Guillerm, J. L. \& Maillet, J. (1982). Western Mediterranean countries from Europe. In: Biology and Ecology of Weeds, (eds Holzner \& Numata), 227-243. The Hague, The Netherlands.

Hoffman, F. (1719). De Millefolio germanice. American Journal Pharmacy. 107:819.

Hoffman L, Fritz D, Nitz S, Kollmannsberger HI, Drawert F (1992) Essential oil composition of three polyploids in the Achillea millefolium "complex". Phytochemistry 31, 537-542.

Kim, H.K., Cheon, B.S., Kim, S.Y. \& Kim, H.P. (1999). Efffects of naturally occurring flavonoids on nitric oxide production in the macrophage cell in RAW 264.7 and their structure-activity relationships. Biochemistry Pharmacology .58, 759-765.

Larson, R.A. (1988). The antioxidants of higher plants. Phytochemistry. 27, 969.978.

Liebman, M.\& Dick, E. (1993). Crop rotation and intercropping strategies for weed management. Ecological Applications. 3, 92-122.

López Mosquera, M.E., Carballo, M.E., Cableiro, F., Carral, E., Lema, M.J., López Fabal, A., Saínz, M.J.(2003). Valorización agronomica de estiércol de pollo deshidratado y granulado en el cultivo de lechuga bajo invernadero. Actas de Horticultura .39, 595-596.

Lourenço, P.M.L., Figueiredo, A.C., Barroso, J.G., Pedro, L.G. Oliveira, M.M., Deans, S.G, \& Scheffer, J.J. (1999). Esential oils from hairy root cultures and from plant roots of Achillea millefolium. Phytochemistry .51, 637-642. 
Maffei, M., Mucciarelli, M.,. \& Scannerini, S. (1994) Essential oils from Achillea Species of different Geographic origin. Biochemical Systematics and Ecology. Vol 22, 7, 679-687.

Maillet, J. (1981). Evolution de la flore adventice dans le Montepelliarais sous la presión des Techniques Culturales. PhD thesis, USTL Montpellier, France.

McCloskey, M.C., Firbank, L.G., Watkinson, A.R. \& Webb, D.J. (1998). Interaction between weeds of winter wheat under different fertilizer, cultivation and weed management treatments. Weeds Research. 38, 11-24.

Mitich, L.W. (1990). Yarrow-The herb of Achilles. Weed Technology. 4, 451-453.

Mitra, B., Saha, A., Chowdhury, A.R., Pal, C., Mandal, S., Mukhopadhyay, S., Bandopadhyay, S. \& Majumder, H.K. (2000). Luteolin an abundant dietary component is a potent antilishmanial agent that acts by inducing to poisomerase 11-meditaded kinetoplast DNA cleavage leading to apoptosis. Molecular Medicine. 6, 527-541.

Pérez-García, F., Adzet, T. \& Canigueral, S. (2000). Activity of artichoke leaf extract on reactive oxygen species in human leukocytes. Free Radical Research .33,661-665.

Pino, J., Sans, F.X. \& Masalles, R.M. (1998). Population dynamics of Rumex obtusifolius under contrasting Lucerne cropping systems. Weed Research. 38, 25-34.

Raunkaier, C. (1905) Types biologiques pour la géographie botanique. Kgl. Danskevidenskaberne selskals Forhanndinger 5, 347-437.

Rice-Evans, C.A., Miller, N.J., \& Paganga, G. (1996). Structure-antioxidant activity relationships of flavonoids and phenolic acids. Free Radical Biolology of Medicine. 27, 1313-1323.

Rohloff, J., Skagen, E.B., Steen, A.H.. \& Iversen, T.H. (2000). Production of yarrow (Achillea millefolium L.) in Norway: essential oil content and quality. Journal of Agricultural and Food Chemistry. 48 (12), 6205-6209.

Scheffer, M.C. \& Ronzeli, P. Jr. (1993). Influence of organic fertilization on the biomass, yield and composition of the essential oil of Achillea millefolium L. Acta Horticuturae. 331, 109-114.

Schulz, H. \& Albroschert, G. (1988). High-performance liquid chromatographic characterization of some medicinal plant extracts used in cosmetic formulas. Journal of chromatography. 442, 353-361.

Sommers, C. (1985). Less tillage cloesn mean more weeds. Successful Farming .84:22-23.
Taleb, A. (1989). Etude de la Flore Adventice des Céréales de la Chaouia (Maroc Occidental). Aspects Botanique, Agronomique et Écologique. Montpellier, France. Thèse DDI, Phytotechnie, France.

Taleb, A.. \& Maillet, J. (1994). Mauvaises herbes des cereals de la Chaouia (Maroc). I. Aspect floristique. Weed Research .34, 345-352.

Veliglu, Y.S., Mazza, G., Gao, L. \& Oomah, B. (1998). Antioxidant activity and total phenolics in selected fruits, vegetables and grain products. Journal of Agricultural and Food Chemistry . 46, 4113-4117.

Verne, G .(1981). Plant Speciation. Columbia University Press, New York.

Vinson, J.A., Hao, Y., Su, X.\& Zubik, L. (1998). Phenol antioxidant quantity and quality in foods :vegetables. Journal of Agricultural and Food Chemistry. 46, 3630-3634.

Vrifsen, R., Everaert, L. \& Boeye, A. (1988). Antiviral activity of flavones and potentiation by ascorbate. Journal of General Virology. 69, 1749. 1751.

Wang H J \& Murphy PA (1994). Isoflavone content in commercial soybean foods. Journal of Agricultural and Food Chemistry, 42:1666-1673.

Weller, S.C. (1986). Weed control considerations in herbs production. In: Proceedings $1^{\text {st }}$ National Herb Growing and Marketing Conference, West Lafayette, Indiana (USA), 9296.

Wen, K.C., Ho, L.K. \& Chang, Y.S. (2000). Analysis of flavonoides and coumarins in Ixeris laevigata var. oldhami by high-performance liquid chromatography. Journal of liquid chromatography and related technologies. 23, 25732583.

Wollenweber, E. (1988). Ocurrence of flavonoid aglycones in medicinal plants. Plant flavonoides in Biology and Medicine II: Biochemical, Cellular and Medicinal Properties, pages 45-55. Alan R., Liss, Inc.

Yoshida, M., Sakai, T., Hosokawa, N., Marui, N. Matsumoto, K.A.., Nishino, H. \& Aoike, A. (1990). The effect of quercetin on cell cycle progression and growth of human gastric cancer cells. FEBS Letter. 260, 10-13.

Zhelezova, S.V. (2001). Dynamics of weed communities in long-term tillage and cropping systems experiment. I World Congress on Conservation Agriculture. Ecaf-FAO. 463-466. 